\title{
Mindfulness and burnout among competitive adolescent tennis players
}

\author{
S P Walker, MSocSc (Couns Psych), PhD \\ Unit for Professional Training and Service in the Behavioural Sciences (UNIBS), Faculty of the Humanities, University of the Free State, \\ Bloemfontein, South Africa
}

Corresponding author: S P Walker (walkersp@ufs.ac.za)

\begin{abstract}
Background. Burnout among adolescent athletes is a cause for concern. However, little is known about the intrapersonal factors that may be related to burnout in this population.

Objectives. To explore the relationship between burnout and mindfulness among competitive adolescent tennis players.

Methods. Competitive adolescent tennis players ( $N=104$; mean age 16 years) completed measures of mindfulness and athlete burnout. Correlations were calculated with regard to mindfulness and burnout. A one-way multivariate analysis of variance (MANOVA) was conducted to determine whether athletes assigned to three levels of mindfulness (high, moderate and low) differed significantly with regard to burnout. Results. Mindfulness exhibited significant negative correlations with global burnout, emotional/physical exhaustion, reduced sense of accomplishment and sport devaluation. The results of the MANOVA indicated that individuals in the three mindfulness groups (high, moderate and low) reported significantly different levels of burnout. Post hoc analyses revealed that participants in the high mindfulness group reported a significantly lower sense of reduced accomplishment and global burnout than participants in the low mindfulness group. In addition, participants in the low mindfulness group reported significantly higher levels of global burnout than individuals in the high and moderate mindfulness groups.

Conclusion. Mindfulness appears to be negatively related to athlete burnout among competitive adolescent tennis players. Furthermore, athletes reporting different levels of mindfulness exhibit differing levels of burnout. The potential protective effect of mindfulness with regard to burnout among adolescent athletes warrants further investigation.
\end{abstract}

S Afr J SM 2013;25(4):105-108. DOI:10.7196/SAJSM.498

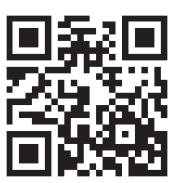

The escalating demands upon athletes competing at all levels of sport are increasingly linked with burnout and fatigue syndromes. ${ }^{[1-3]}$ Athlete burnout may be viewed as the long-term consequence of an imbalance between an athlete's coping resources and the physical, social and psychological stress that they are exposed to on an ongoing basis through training and competition. In general, burnout is characterised by: (i) physical and emotional exhaustion; (ii) a reduced sense of accomplishment, most commonly manifested in reduced physical performance or the subjective perception that the athlete is no longer able to reach their specific performance goals; and (iii) the tendency to disinvest from, or to devalue participation in the chosen sport. ${ }^{[4]}$ Burnout has been linked to a number of negative outcomes among athletes, including reduced motivation, dysfunctional coping behaviour, depression, suboptimal response to training and withdrawal from competitive sport. ${ }^{[1,5-6]}$ However, despite the realisation that athlete burnout is a significant problem, very little research has directly addressed burnout among adolescent athletes. ${ }^{[6]}$ This is of particular concern given the association between athlete burnout and withdrawal from competitive sport among adolescents. ${ }^{[1,2]}$ There is thus a need to develop a better understanding not only of the factors that contribute to burnout among adolescent athletes, but also of intrapersonal factors that may play a protective role in this regard.
Mindfulness may potentially serve a protective function with regard to athlete burnout. Mindfulness has been described as the non-judgemental awareness of internal and external experiences as they occur in the present moment. ${ }^{[7]}$ Mindfulness has its basis in Eastern meditation practice and has been positively associated with psychological well-being. ${ }^{[7]}$ Recently, mindfulness-based techniques and approaches have been introduced into Western psychology through various stress-management programmes and cognitive-behavioural therapy modalities. These approaches have been shown to have a number of benefits including lower levels of anxiety and depression, as well as increased stress tolerance and improved psychological wellbeing. ${ }^{[8]}$ Mindfulness has also been successfully applied to the treatment of various psychological and psychosocial disorders. ${ }^{[9,10]}$ In addition, mindfulness has been demonstrated to be effective in the promotion of health behaviours such as the initiation and maintenance of physical exercise regimens. ${ }^{[1]}$ To date, however, most studies on mindfulness have focussed on the effect of mindfulness-based interventions on the well-being of individuals, rather than exploring the possible protective effect that existing levels of mindfulness may have when individuals are confronted with stressful life events.

The vast majority of research on mindfulness in sport psychology has focused on the role of mindfulness-based interventions on sporting performance. ${ }^{[12,13]}$ To date, only one study seems to have focused on 
the efficacy of mindfulness as a means of treating athlete burnout. This case study reports a significant reduction in burnout symptoms, as well as an increase in subjective well-being and athletic performance in an Olympic shotist. ${ }^{[14]}$ However, the possible protective role of mindfulness with regard to athlete burnout does not appear to have been investigated, nor the relationship between mindfulness and burnout, among adolescent athletes. Consequently, the objective of the current study was: (i) to determine whether a relationship exists between mindfulness and burnout among competitive adolescent tennis players; and (ii) if so, to determine whether adolescent tennis players with differing levels of mindfulness report different levels of burnout.

\section{Methods}

\section{Participants}

Ethical clearance to conduct the study was obtained from the relevant institutional body. The organisers of two national tennis tournaments gave permission for data to be collected. Only tennis players aged 14 - 19 years were invited to participate in the study. Informed consent was obtained from all participants, as well as from their guardians, prior to administration of the questionnaires. Participants completed the measures listed below between matches or at the end of the day. One hundred and thirteen adolescents participated in the study. Nine participants were excluded due to incomplete questionnaires. The mean age of the final sample $(n=104)$ was 16 years (standard deviation $(\mathrm{SD}) \pm 1$ ). The sample was evenly split with regard to gender, and participants reported having received a mean 6.3 years $(\mathrm{SD} \pm 2.7)$ of professional tennis coaching. The majority of participants (37\%) reported provincial tournaments as their highest level of competition, while $28 \%$ competed at national level, $12 \%$ at regional level and $23 \%$ represented their schools.

\section{Measures}

The Athlete Burnout Questionnaire (ABQ) was used to measure burnout. ${ }^{[4]}$ The ABQ is a 15 -item self-report questionnaire that yields scores on three subscales: reduced sense of sport accomplishment; sport devaluation; and emotional/physical exhaustion. A total burnout score is also derived. Responses to each item are indicated along a fivepoint Likert-type scale anchored by 'almost never' and 'almost always'. Higher scores are indicative of higher levels of burnout. The ABQ has been demonstrated to have acceptable construct validity and internal consistency. ${ }^{[4]}$ Internal consistency was also found to be acceptable in the current sample ( $\alpha=0.710-0.917)$.
The Freiburg Mindfulness Inventory (FMI) was used as a measure of mindfulness. ${ }^{[15]}$ The FMI is a 14 -item self-report questionnaire, with responses indicated along a four-point Likert-type scale anchored by 'rarely' and 'almost always'. A unitary mindfulness score is derived. Higher scores indicate higher levels of mindfulness. A statisticallyderived eight-item version of the FMI has also been proposed. The shorter version of the questionnaire has been shown to be a reliable and valid measure of mindfulness. ${ }^{[15]}$ In the interests of reducing respondent fatigue, the shorter FMI was employed in this study. The eight-item version FMI exhibited an acceptable level of internal reliability in the current sample ( $\alpha=0.703)$.

The ABQ and FMI were translated into Afrikaans using backtranslation. ${ }^{[16]}$ Participants thus had the option of completing the questionnaires in either English or Afrikaans.

\section{Analysis}

Pearson's correlation coefficients were calculated with regard to mindfulness (FMI total score) and burnout (three ABQ subscales and total score). In addition, participants were divided into three levels of mindfulness (high, moderate and low) based on the distribution of the FMI scores in the sample. A one-way multivariate analysis of variance (MANOVA) was then conducted to determine whether individuals in the three levels of mindfulness differed significantly with regard to their ABQ subscale and total mean scores. Post hoc analyses (Scheffé test) were conducted to determine the nature and direction of the significant differences yielded by the MANOVA.

\section{Results}

The correlations between the FMI and ABQ scores for the sample are provided in Table 1. The mean scores, SDs and internal consistencies for each of the scales are also reported. It is apparent that all the measures utilised in the study exhibited acceptable levels of internal consistency; they may thus be included in further analyses. ${ }^{[17]}$ The correlation coefficients reported above suggest that mindfulness is negatively significantly correlated with all three ABQ subscales (reduced sense of accomplishment (-RA): $p \leq 0.01$; devaluation (-D): $p \leq 0.01$; and emotional/physical exhaustion (-E): $p \leq 0.05)$, as well as with total burnout $(p \leq 0.01)$. It would thus appear that higher levels of mindfulness are significantly associated with lower levels of athlete burnout in the current sample.

Given that a significant inverse relationship exists between mindfulness and burnout, it was decided to investigate whether individuals

Table 1. Pearson correlation coefficients, means, SDs, ranges and internal consistencies for the FMI and ABQ

\begin{tabular}{|c|c|c|c|c|c|c|c|c|}
\hline & ABQ-RA & ABQ-D & ABQ-E & Total & FMI & Mean $( \pm S D)$ & Range & $\alpha$ \\
\hline FMI & $-0.406^{*}$ & $-0.300^{*}$ & $-0.233^{\dagger}$ & $-0.354^{*}$ & - & $23.25( \pm 3.87)$ & $14-32$ & 0.703 \\
\hline Total & $0.756^{*}$ & $0.944^{\star}$ & $0.878^{\star}$ & - & & $35.95( \pm 12.41)$ & $15-68$ & 0.917 \\
\hline ABQ-E & $0.443^{\star}$ & $0.774^{\star}$ & - & & & $11.37( \pm 5.07)$ & $5-25$ & 0.888 \\
\hline$A B Q-D$ & $0.633^{\star}$ & - & & & & $11.74( \pm 5.42)$ & $5-25$ & 0.883 \\
\hline ABQ-RA & - & & & & & $12.85( \pm 3.76)$ & $5-20$ & 0.710 \\
\hline \multicolumn{9}{|c|}{${ }^{*} p \leq 0.01 ;{ }^{\dagger} p \leq 0.05}$. \\
\hline
\end{tabular}


reporting different levels of mindfulness differed significantly with regard to the levels of burnout that they experienced. Consequently, three levels (high, moderate and low) of the independent variable (mindfulness) were created by dividing the sample into thirds based on the distribution of their FMI scores (low: FMI $\leq 21 ; n=36$; moderate: FMI 22 - 25; $n=41$; high: FMI $\geq 26 ; n=27)$. It should be noted, however, that mean ABQ scores reported in Table 1 are not particularly high. Consequently, the individuals in this study do not appear to be suffering from significant burnout. To control for the possible effect of gender and level of competition on mindfulness group membership, Pearson's $\chi^{2}$ tests were conducted. The results indicated a proportional distribution across the three levels of the independent variable (levels of mindfulness $)$ when gender $\left(\chi^{2}=1.395 ; d f=2 ; p=0.498\right)$ and level of competition $\left(\chi^{2}=12.527 ; d f=6 ; p=0.051\right)$ were taken into account. It can therefore be assumed that individuals of both genders and all four levels of competition were proportionally and equally distributed across the three mindfulness groups. Consequently, any differences in levels of burnout between these three groups could not be attributed to the effect of gender or level of competition.

A one-way between-groups analysis of variance (ANOVA) was conducted to investigate differences between the three levels (high, moderate and low) of the independent variable (mindfulness) with regard to athlete burnout. Four dependent variables were included in the analysis: reduced sense of accomplishment (RA), devaluation (D), emotional/physical exhaustion (E) and total burnout (total). Preliminary assumption testing was conducted to check for normality, linearity, univariate and multivariate outliers, homogeneity of variancecovariance matrices and multicollinearity. Violations of the assumptions of homogeneity of variance-covariance were detected. Consequently, a Bonferroni adjusted $\alpha$-level of 0.013 was used to determine statistical significance in the ensuing analyses. A statistically significant difference was apparent between the levels of mindfulness on the combined dependent variables $(F(6 ; 198)=3.7441 ; p=0.001$; Wilks' $\lambda=0.807$; partial $\left.\eta^{2}=0.102\right)$. Follow-up univariate ANOVAs were conducted to ascertain the specific $A B Q$ subscales with regard to which the three burnout groups differed (Table 2 ).

Significant $(p \leq 0.013)$ differences were apparent for the level of mindfulness with regard to reduced sense of accomplishment (ABQ$\mathrm{RA} ; p=0.000$ ), devaluation (ABQ-D; $p=0.002$ ), emotional/physical burnout (ABQ-E; $p=0.011$ ) and total burnout (ABQ total; $p=0.000$ ) (Table 2). The corresponding $f$-values suggested that these results were indicative of large effect sizes with regard to reduced sense of accomplishment $(f=0.160)$ and total burnout $(f=0.145)$, and medium effect sizes with regard to devaluation $(f=0.117)$ and emotional/physical exhaustion $(f=0.085)$. Consequently, these findings can be considered to be of moderate to significant practical importance. ${ }^{[18]}$ Individuals in the three mindfulness groups thus reported levels of reduced sense of accomplishment, devaluation, physical/emotional exhaustion and total burnout that differed to a statistically and practically significant degree.

Post hoc comparisons using the Scheffé test indicted that the mean ABQ-RA score for the high FMI group (mean 10.74; $\mathrm{SD} \pm 4.09$ ) was significantly lower $(p=0.000)$ than that of the low FMI group (mean 14.61; $\mathrm{SD} \pm 2.95)$. Similarly, the mean ABQ-D score for the high FMI group (mean 9.88; $\mathrm{SD} \pm 6.19$ ) was significantly lower $(p=0.005)$ than that of the low FMI group (mean 14.22; SD \pm 5.16 ). Furthermore, the mean $\mathrm{ABQ}$ total score for the low FMI group (mean 42.22; $\mathrm{SD} \pm 10.53$ ) was significantly higher than the mean $\mathrm{ABQ}$ total scores of both the moderate FMI (mean 33.76; $\mathrm{SD} \pm 10.23 ; p=0.008$ ) and high FMI (mean $30.93 ; \mathrm{SD} \pm 14.56 ; p=0.001$ ) groups. The $\mathrm{ABQ}$ total means for the high and moderate FMI groups, however, did not differ significantly. No significant differences were found between the three mindfulness groups with regard to their mean ABQ-E scores.

\section{Discussion}

The results of the current study indicate that mindfulness is significantly and inversely related to physical and emotional exhaustion, a reduced sense of sporting accomplishment, sport devaluation and global athletic burnout among competitive adolescent tennis players. High levels of mindfulness are thus associated with lower levels of burnout in this group of athletes. These findings appear to be in keeping with much of the existing literature on mindfulness in sport. ${ }^{[14]}$ Higher levels of mindfulness have also been associated with increased focus, more vivid imagery and improved sporting performance. ${ }^{[11,19]}$ Mindfulness would thus appear to be related to a number of favourable outcomes among athletes, including reduced burnout symptomatology. It could be hypothesised from a theoretical perspective that by maintaining an open and non-judgemental orientation towards their experiences in the present moment, athletes with higher levels of mindfulness are less likely to engage in critical self-evaluation or repetitive thought processes often associated with increased emotional distress and reduced satisfaction with their general level of sporting achievement. ${ }^{[1,2,7]}$

The results of the ANOVAs revealed that adolescent tennis players reporting different levels of mindfulness also reported differing levels of burnout. More specifically, the high mindfulness participants reported significantly lower burnout than the moderate and low mindfulness participants. Adolescent tennis players displaying low

Table 2. Means, SDs and F-values for the one-way analysis of variance (ANOVA) for the three levels of mindfulness

\begin{tabular}{|c|c|c|c|c|c|c|}
\hline ABQ scale & $\begin{array}{l}\text { High FMI } \\
\text { mean }( \pm \mathrm{SD})\end{array}$ & $\begin{array}{l}\text { Moderate FMI } \\
\text { mean }( \pm \mathrm{SD})\end{array}$ & $\begin{array}{l}\text { Low FMI } \\
\text { mean }( \pm \mathrm{SD})\end{array}$ & $F$-value & $p$-value & $f$-value \\
\hline ABQ-RA & $10.74( \pm 4.09)$ & $12.68( \pm 3.48)$ & $14.61( \pm 2.95)$ & $9.605^{\star}$ & 0.000 & 0.160 \\
\hline ABQ-D & $9.88( \pm 6.19)$ & $10.78( \pm 4.30)$ & $14.22( \pm 5.16)$ & $6.664^{*}$ & 0.002 & 0.117 \\
\hline ABQ-E & $10.30( \pm 6.02)$ & $10.29( \pm 4.25)$ & $13.39( \pm 4.66)$ & $4.692^{*}$ & 0.011 & 0.085 \\
\hline Total & $30.93( \pm 14.56)$ & $33.76( \pm 10.23)$ & $42.22( \pm 10.53)$ & $8.538^{\star}$ & 0.000 & 0.145 \\
\hline
\end{tabular}


levels of mindfulness reported a significantly higher sense of reduced sporting accomplishment and significantly higher sport devaluation than those with high levels of mindfulness. As noted earlier, an increased tendency to engage in critical self-evaluation and rumination with regard to the pace of their sporting progress may incline low mindfulness athletes towards a reduced sense of accomplishment and consequent devaluation of their sport participation. Adolescent tennis players reporting low levels of mindfulness were also found to have significantly higher global athlete burnout scores than the moderate and high mindfulness participants. The tentative conclusions that could be drawn from these findings are that not only may higher levels of mindfulness be desirable due to an inverse relationship with burnout, but lower levels of mindfulness may be undesirable due to a significantly stronger association with global burnout than with moderate or high levels of mindfulness.

No significant differences were found between the three mindfulness groups concerning physical and emotional exhaustion. This may be indicative of the phase of the playing careers in which the participants currently find themselves. It is possible that physical and mental exhaustion are not prominent burnout symptoms among adolescents who are still developing technically as athletes. The prominent areas of focus, and thus also the most probable areas of burnout presentation, may be related more to issues of developing technical competence, reaching certain performance goals and viewing one's level of achievement positively in comparison to that of one's peers. ${ }^{[1,2]}$ Consequently, individuals in this phase of their athletic development may be more inclined to experience burnout in terms of a reduced sense of accomplishment and sport devaluation than in terms of physical and emotional exhaustion.

\section{Study limitations}

This research is not without limitations. First, a cross-sectional correlational design was employed, thus no conclusions can be drawn regarding possible causal relationships between mindfulness and burnout. Future longitudinal and intervention-based research would be valuable in establishing the causal nature of the relationship between these two variables. More sophisticated models should be developed to establish the pathways of causality between mindfulness and burnout, as well as the possible role of other cognitive and emotional mechanisms in the experience of burnout among adolescent athletes. Second, the study made use of a homogenous and relatively small sample. The findings can thus not be generalised beyond the current sample. Third, athletes in the current sample did not display particularly high levels of global burnout (mean score 23.25). Consequently, the results cannot be generalised to athletes exhibiting high levels of burnout. Future research would do well to focus specifically on athletes reporting high levels of burnout, as well as on individuals who have withdrawn from competitive sport due to burnout. Finally, the FMI is generally employed to measure mindfulness among adults. Consequently, this measure may not provide as valid a measure of mindfulness among adolescents. Future studies employing more adolescent- and sportspecific measures of mindfulness appear to be warranted.

\section{Conclusion}

While largely exploratory in nature, this study highlighted the association between mindfulness and burnout among adolescent tennis players. It would appear that adolescent tennis players with high levels of mindfulness are inclined to report significantly fewer symptoms of burnout than those with low levels of mindfulness. Further, those players reporting low levels of mindfulness appear to be significantly more inclined to experience a reduced sense of accomplishment with regard to their sporting pursuits, and are more inclined to devalue their sport participation. Notwithstanding the limitations of the current study and the obvious need for further research, there would appear to be merit in promoting mindfulness among adolescent athletes. Coaches and performance consultants should consider introducing mindfulness interventions as a means of reducing burnout risk in this population.

Acknowledgements. The author is indebted to Ms Hanli du Toit for her assistance with data collection and Prof. Karel Esterhuyse for his comments on earlier drafts of this manuscript.

\section{References}

1. Fraser-Thomas J, Côté J, Deakin J. Understanding dropout and prolonged engagement in adolescent competitive sport. Psychol Sport Exerc 2008;9(5):645-662. [http://dx.doi.org/ 10.1016/j.psychsport.2007.08.003]

2. Harris BS, Watson JC. Assessing youth sport burnout: A self-determination and identity development perspective. J Clin Sport Psychol 2011;5(2):117-133.

3. Gustafsson H, Kenttä G, Hassmén P, Lundqvist C. Prevalence of burnout in competitive adolescent athletes. Sport Psychologist 2007;21(1):21-37.

4. Raedeke TD, Smith AL. Development and preliminary validation of an athlete burnout measure. J Sport Exerc Psychol 2001;23(4):281-306.

5. Gustafsson H, Kenttä G, Hassmén P. Athlete burnout: An integrated model and future research directions. Int Rev Sport Exerc Psychol 2011;4(1):3-24. [http://dx.doi. org/10.180/1750984X.210.541927]

6. Goodger K, Gorely T, Lavallee D, Harwood C. Burnout in sport: A systematic review. Sport Psychologist 2007;21(2):127-151.

7. Baer RA. Mindfulness training as a clinical intervention: A conceptual and empirical review. Clin Psychol Sci Prac 2003;10(2):125-143. [http://dx.doi.org/10.1093/clipsy. bpg016]

8. Carmody J, Baer RA. Relationships between mindfulness practice and levels of mindfulness, medical and psychological symptoms and well-being in a mindfulness-based stress reduction program. J Behav Med 2008;31(1):23-33. [http://dx.doi.org/10.1007/ s10865-007-9130-7]

9. Arch JJ, Ayers CR, Baker A, et al. Randomized clinical trial of adapted mindfulnessbased stress reduction versus group cognitive behavioural therapy for heterogeneous anxiety disorders. Behav Res Ther 2013;51(4-5):185-196. [http://dx.doi.org/10.1016/j. brat.2013.01.003]

10. Cassidy EL, Atherton RJ, Robertson N, Walsh DA, Gillet R. Mindfulness, functioning and catastrophizing after multidisciplinary pain management for chronic low back pain. Pain 2012;153(3):644-650. [http://dx.doi.org/10.1016/j.pain.2011.11.027]

11. Ulmer CS, Stetson BA, Salmo PG. Mindfulness and acceptance are associated with exercise maintenance in YMCA exercisers. Behav Res Ther 2010;48(8):805-809. [http:// dx.doi.org/10.1016/j.brat.2010.04.009]

12. Aherne C, Moran AP, Lonsdale C. The effect of mindfulness training on athletes' flow: An initial investigation. Sport Psychologist 2011;25(2):177-189.

13. Schwanhausser L. Application of the mindfulness-acceptance-commitment (MAC) protocol with an adolescent springboard diver. J Clin Sport Psychol 2009;3(4):377-395.

14. Jouper J, Gustafsson H. Mindful recovery: A case study of a burned out elite shooter. Sport Psychologist 2013;27(1):92-102.

15. Kohls N, Sauer S, Walach H. Facets of mindfulness - results of an online study investigating the Freiburg Mindfulness Inventory. Pers Indv Diff 2009;46(2):224-230. [http://dx.doi. org/10.1016/j.paid.2008.10.009]

16. Brislin RW. Back-translation for cross-cultural research. J Cross-Cultural Res 1970;1(3):185-216.

17. Foster JJ, Parker I. Carrying Out Investigations in Psychology: Methods and Statistics. Leicester: The British Psychological Society, 1999.

18. Cohen J. Statistical Power Analysis for the Behavioural Sciences. 2nd ed. New York: Academic Press, 1988.

19. Thompson RW, Kaufman KA, De Petrillo LA, Glass CR, Arnkoff DB. One year followup of mindful sport performance enhancement (MSPE) with archers, golfers and runners. J Clin Sport Psychol 2011;5(2):99-116. 